\title{
Investigation of The Nucleation Process of Electrodeposited Nanostructured Cobalt Films Using Brij 76 Lyotropic Liquid Crystal
}

\author{
Ahmed A. Al Owais ${ }^{1}$ and Ibrahim S. El-Hallag ${ }^{2}$ \\ ${ }^{1}$ Chemistry Department, College of Science, King Saud University, Riyadh, Saudi Arabia \\ ${ }^{2}$ Chemistry Department, Faculty of Science, Tanta University, Tanta, Egypt
}

Corresponding Author Email: i.elhallag@yahoo.com

\begin{abstract}
In this article we describe uncomplicated and flexible method for the preparation of three-dimensional highly ordered mesoporous cobalt films containing close arranged arrays of spherical void of uniform size. Arranged mesoporous cobalt films were precisely prepared by electrodeposition of hexagonal H1-e Co ion using Brij -76 template. The electrodeposited mesopores films were described via various techniques such as electronic microscopy (SEM \&TEM), low angle X-ray diffraction (XRD), cyclic voltammetric and chronoamperometric methods. The appropriateness of thin films as high - performance super capacitors electrode materials is indicated electrochemically using cyclic voltammetry $(\mathrm{CV})$ technique. The initial stages of nucleation, growth mechanism of electrodeposition of Co was demonstrated via cyclic voltammetry and chronoamperometry tools.
\end{abstract}

Keywords: nanostructured, mesoporous, electrodeposition, Brij76, cyclic voltammetry, nucleation

Received: March-16-2020, Accepted: April-20-2020, https://doi.org/10.14447/jnmes.v23i2.a10

\section{INTRODUCTION}

Mesoporous materials exhibit very interesting electrical, optical, magnetic and chemical properties, which could not be achieved by their bulk counterparts [1]. Mesoporous materials may also be used in medical imaging, drug targeting and other biological applications and catalysis [2]

Interest in electrochemical capacitors (ECs) has been soared newly due to their higher power density and longer cycle life compared to secondary batteries and great energy density that conventional electrical double-layer capacitors [3-5]. They play increasingly important roles in power source applications such as auxiliary power sources for mobile electronic devices, etc. $[6,7]$. Electrochemical capacitors employ both electrical double layer and interfacial redox processes to pack energy at an electrode/electrolyte interface. Accordingly, structural properties are strongly related to the electrochemical properties of Co films for supercapacitors. It was established that, the pore size at the range of 2-50 nm (mesopores) is treated to be greatly desirable for the electrochemical super capacitors, and the ordered mesoporous make ionic motion easier compared with conventional mesoporous material in which the pores are randomly connected [8].

The hexagonal phase composition of the Brij $76 / \mathrm{Co} / \mathrm{p}$ xylene system was determined according to the phase diagram established in literature Raman et al. [9-11]. The fabrication of nanostructured materials using surfactant molecules as templates was established to be a very effective area of research [12, 13]. In the hexagonal phase the surfactant molecules collect into long, cylindrical micelles and these micelles then compact into a hexagonal group (Figure 1) in which the separation between the micelles is comparable to their diameter (around $2 \mathrm{~nm}$ ) [14]. When these phases are employed to template the electrochemical deposition of metal layers, the metal salt and electrolyte are dissolved into the aqueous constituents of the mixture and metal deposition take place from the electrode surface, around the surfactant micelles. When deposition is completely finish, the surfactant is removed by washing to leave a metal film punctured by a regular hexagonal array of uniform pores. The thickness of the film is directly governed by the total charge transferred.

The nucleation of the metal state and the increase of the number of nuclei are indicated with only a slight maximums in the i-t plots. The representative maximums indicate the mechanism of nucleation and the slow increase of the current up to a certain value suggest a three-dimensional growth of the nuclei [11].

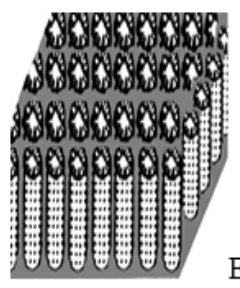

Electrodeposition of cobalt ions.

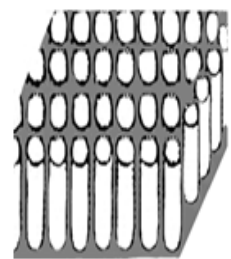

Remove template

Figure 1. Indicate the growth process of the H1-e Co films in the presence of the hexagonal liquid crystal template.

The described work explains the fabrication of nanostructured mesoporous cobalt films (H1-eCo) by the electrodeposition of cobalt from the aqueous area of the hexagonal liquid crystal phase of Brij 76. The structure of these $\mathrm{H}_{1}$-eCo films has been examined using scanning electron microscopy (SEM), transmission electron microscopy (TEM) and_X-ray diffraction. Electrochemical capacitance performance of the films was investigated by the means of cyclic voltammetry technique (CV). Also, to obtaining some data about the general mechanism of deposition of $\mathrm{Co}$ in 
Brij76 template in slightly acidic acetate electrolyte, as well as to obtain information about the initial stages of deposition of Co, cyclic voltammetry and chronoamperometry methods were used.

\section{EXPERIMENTAL}

\subsection{Chemicals and Materials}

The surfactants polyoxyethylene (10) stearyl ether (Brij-76) has structural formula $\mathrm{C}_{18} \mathrm{H}_{37}\left(\mathrm{OCH}_{2} \mathrm{CH}_{2}\right)_{10} \mathrm{OH}$, was purchased from Aldrich. The solutions and liquid crystalline template (LCT) used were prepared using reagent grade. Also, The glassware was cleaned by immersing in 5\% Decon 90 (Aldrich solution for a few days followed by flushing with deionized water and dried in an oven at $50^{\circ} \mathrm{C}$. P-xylene $(99 \%)$, cobalt acetate (CoAc2.4H2O 99.5\%), potassium acetate (KAc 99\%) and boric acid $\left(\mathrm{H}_{3} \mathrm{BO}_{3}\right)$ were obtained from Aldrich. The plating mixtures used in this work was ternary system consisting Brij 76 non-ionic surfactant $\left(\mathrm{C}_{20} \mathrm{H}_{42} \mathrm{O}_{2}\right)$, aqueous solution of $\mathrm{CoAc}_{2} .4 \mathrm{H}_{2} \mathrm{O}, \mathrm{H}_{3} \mathrm{BO}_{3}$ and p-xylene. The $\mathrm{H}_{1}$ phase of ternary mixture containing $53.13 \mathrm{wt} \%$ aqueous Co salts and boric acid, $44.6 \%$ weight of Brij 76 and $2.23 \%$ weight of $p$ xylene which is firm at room temperature and still chemically stable for more than a month. Electrodeposition from the liquid crystalline plating mixture onto a polished gold electrode was performed at room temperature, after deposition the electrodes were flushed with extra amounts of deionized water to get rid of the surfactant. The gold electrode was cleaned by sonication in propanol (BDH) for $1 \mathrm{~h}$ followed by rinsing with deionized water [15].

\subsection{Instrumentation}

The electrochemical experiments were conducted using an EG \& G 283 Potentiostat and an ordinary three electrode cell (15 mL volume capacity). In electrochemical measurements, the working electrode was a $1 \mathrm{~mm}$ diameter gold disk electrode, while for the structural characterization of the produced material films, the working electrode was $1 \mathrm{~cm}^{2}$ are a flat electrode. Platinum gauze of $1 \mathrm{~cm}^{2}$ area was utilized as counter electrode and saturated calomel electrode (SCE) as the reference electrode. $1 \mathrm{~mm}$ disk electrode was polished by polishing paper (grade 1200) followed by alumina (Buehler) of two grades: 1.0 and $0.3 \mu \mathrm{m}$ then rinsed with deionized water. An analytical scanning electron microscope (JOEL 6400) was used to exhibits the morphology and depth of the electrodeposited cobalt films. Samples for SEM analysis were prepared by electroplating Co from the template mixture on gold electrodes sheets (area $1 \mathrm{~cm}^{2}$ ). These gold electrodes were cleaned up in an ultrasonic bath of 2-propanol for $10 \mathrm{~min}$ before the experiment. XRD with $\mathrm{Cu} \mathrm{K} \alpha$ radiation was used to prove primary evidence for the formation of a nanostructured film. The regularity of the nanostructure was investigated using JEOL 2000FX transmission electron microscope operating at an accelerating voltage of $200 \mathrm{KV}$. The nanostructured Co samples were firmly adhered on the gold electrode surface, and samples for TEM analysis was prepared by scraping small samples of cobalt off of the gold electrode surface and on to the TEM grid using a scalpel.

\section{RESULTS AND DISCUSSION}

\subsection{Structural Analysis}

Polarized-light optical microscopy (POM) and low-angle XRD were used before electrodeposition for determination of the electrolyte phase structure. Figure 2 shows the typical POM image for Brij76 templated Cobalt. The figure indicates that, the spacing between the three axes in the same plane equal $120^{\circ}$ which confirming the formation of hexagonal structure [16].

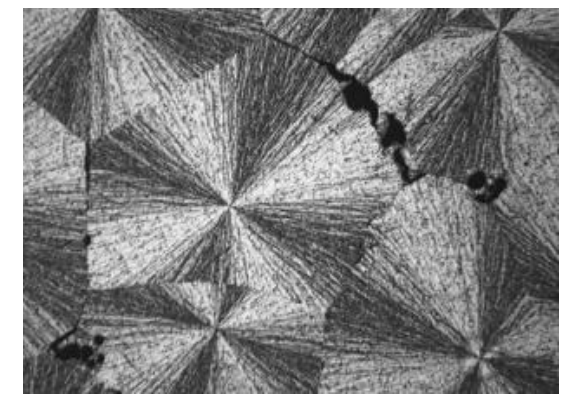

Figure 2. Image of polarized-light optical microscopy for Brij76 templated CoAc2

\subsection{SEM and TEM of cobalt/Brij 78 films}

The nanoarchitecture of the final electrochemically deposited film is determined by the structure of the lyotropic phase used of deposition [10-12, 17]. The cobalt films which were electrochemically deposited at $-0.92 \mathrm{~V} v s$. SCE from the hexagonal phase template mixture have a shiny gray color silvery metallic luster and adhered well to the evaporated gold electrodes used for the deposition. Figure 3A exhibits scanning electron micrograph of an $\mathrm{H}_{1}$-eCo film which displays the cobalt film formed is featureless.

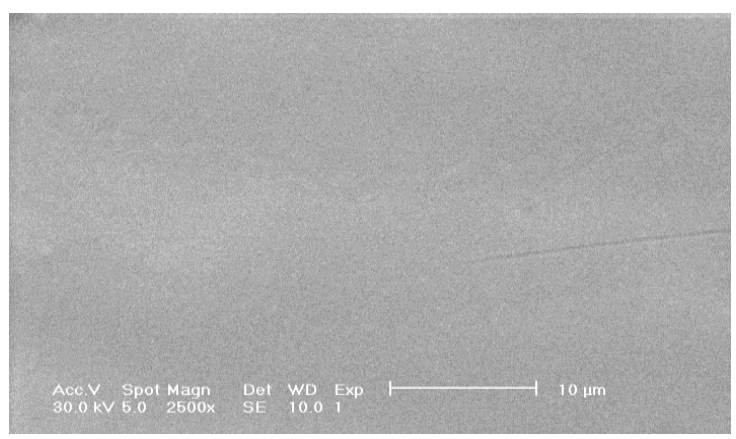

(A)

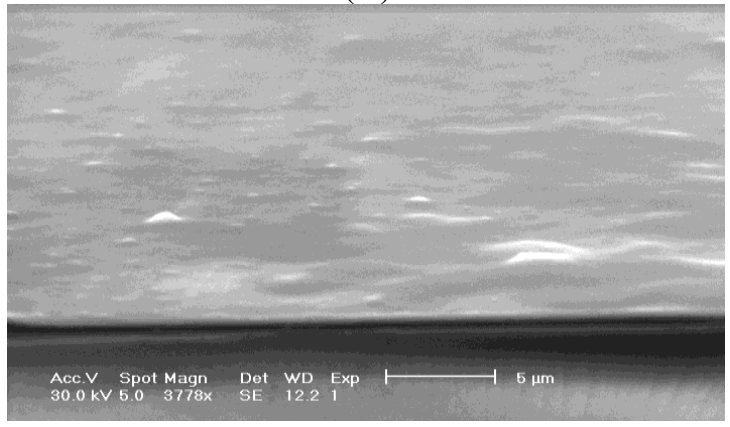

(B)

Figure 3. SEM of H1-e Co films in the presence of a hexagonal liquid crystal template (A), Cross-section view of Co film (B) 


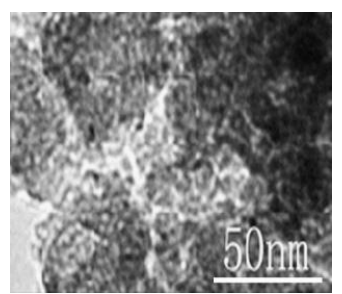

Figure 4. TEM images of the deposited cobalt at potential $-0.92 \mathrm{~V}$

The cross-sectional view (Figure 3B) shows that the film is compact, smooth and uniform. The film is about $219 \mathrm{~nm}$ thick. There is no evidence for nanostructure visible on the scale of the SEM. To examine the nanostructure of the electrochemically deposited cobalt films, it is necessary to use TEM as shown in Figure 4.

It is apparent that the TEM images have orderly and well arranged hexagonal arrays of mesoporous structure. The bright areas correspond to the pores left after deportation of the surfactant template from the electrochemically deposited films. The dark areas correspond to the electrodeposited cobalt metal. From the images it is clear that the pores are hexagonally arranged over length scales. Inspections of these pores show that they are continuous and approximately straight over their whole length, although some defects are evident. These defects and the minor deviation from linearity are normal if the cobalt is a cast of the lyotropic crystalline phase when it is a soft solid [18, 19]. The films are estimated to have a pore center to center distance of about $10.14 \mathrm{~nm}$, with a uniform pore diameter of about $9.2 \mathrm{~nm}$ and cobalt wall thickness of about $5.4 \mathrm{~nm}$. According to the TEM results, it is noted that the mesostructures of the $\mathrm{H}_{1}$-eCo almost remain unchanged under different deposition potentials, which is consistent with the results reported in literature [20-23]. On the basis of the pore size and wall thickness, a high specific surface area inside these pores would be expected. This is supported by X-ray investigation.

\subsection{X-ray characterization of $\mathrm{H}_{1}$-eCo films.}

Figure 5 shows the low-angle XRD patterns of the Brij76 templated Co electrolyte from CoAc2, which exhibits hexagonal symmetry with $d$ spacing of $7.53 \mathrm{~nm}$ and $4.83 \mathrm{~nm}$ for (100) and (110) reflections. As shown, a well-known higher intensity peak corresponding to a d-spacing of $7.53 \mathrm{~nm}$ is exhibited by the low angle XRD pattern for the electrodeposited cobalt. This is consistent with the deposition of a cobalt film with a porous nanostructure obtained from the structure of the template mixture. However, the ordering of $\mathrm{H}_{\mathrm{I}}-\mathrm{Co}$ film is less than the hexagonal liquid crystalline phase of the template mixture as indicating by the absence of higher diffraction planes and the width of $\mathrm{H}_{\mathrm{I}}$-Co at half maximum is much larger than the width of (100) the plane peak of the template mixture.

The pore -to- pore distance (r) for these hexagonal arrays given by:

$$
\mathrm{r}=\mathrm{d}_{110} / \cos 30
$$

is $8.54 \mathrm{~nm}$. The basic vector length (lattice parameter), $a_{h}$ of the hexagonal phases can be obtained by using the following relation $[21]$ :

$$
a_{h}=(2 d / \sqrt{ } 3)
$$

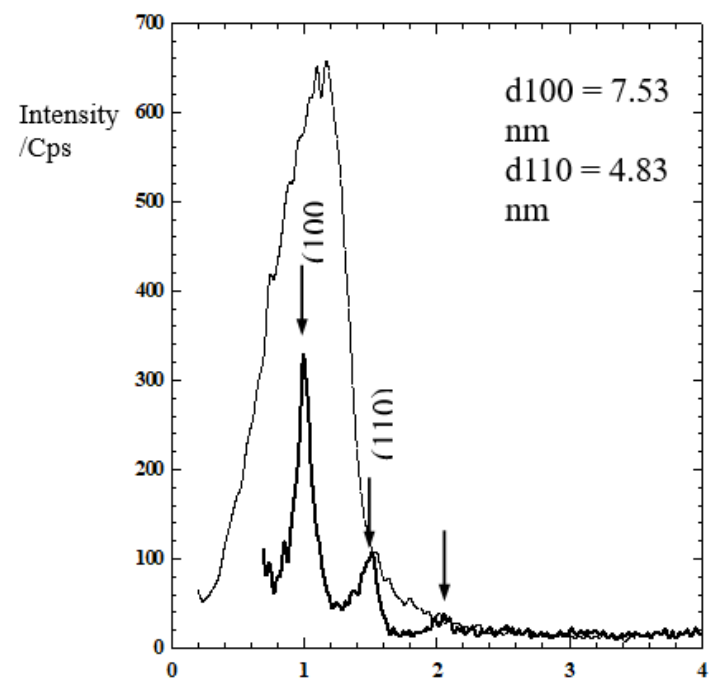

Figure 5. Low angle XRD (-) for the

hexagonal lyotropic $\left(\mathrm{H}_{\mathrm{I}}\right)$ liquid crystalline phase of the template mixture, Low angle XRD $(-)$ for a nanostructured

$\mathrm{H}_{\mathrm{I}}$-eCo film deposited from the haxagonal phase of the Brij ${ }^{\circledR} 76$ template mixture

As calculated from Eq. (2) the distance between two centers of adjacent cylinders, $\mathrm{a}_{\mathrm{h}}$ for the pure hexagonal phase is 8.17 $\mathrm{nm}$ agrees well with the value calculated from Eq. (1). The appearance of low angle peal indicates that mesoscopic order in the liquid crystalline template is preserves in the nanoporous Co films. The broading of the single films diffraction peak may be due to a partial destruction of long-range order during electrodeposition.

\subsection{Cyclic voltammetry investigation.}

In this work, the electrochemical characteristics of electrodeposited cobalt film were investigated by cyclic voltammetry technique in $0.1 \mathrm{M} \mathrm{NaOH}$ at various scan rates. Figure 6 exhibits the characteristic $\mathrm{CV}$ curves of the cobalt film electrode at two different scan rates. It can be noticed that the plot exhibits a couple of peaks corresponding to the redox reaction of cobalt film [23].

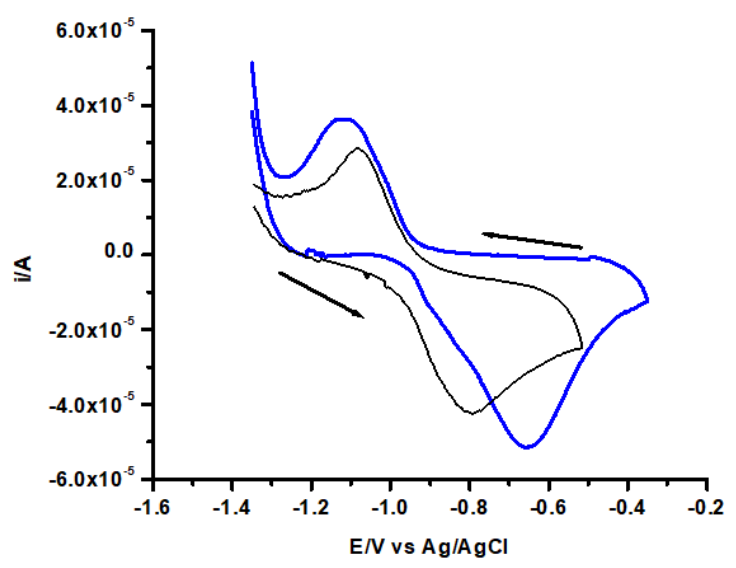

Figure 6. Display a two cyclic voltammograms of cobalt film in $0.1 \mathrm{M} \mathrm{NaOH}$ at two different scan rates $\left(0.3 \mathrm{Vs}^{-1}\right.$ blue line and $0.2 \mathrm{Vs}^{-1}$ black line) 


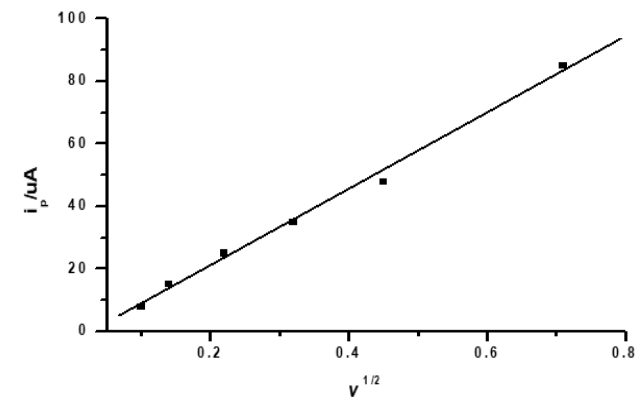

Figure 7. Shows a linear dependence of $i_{p}$ on $v^{1 / 2}$ of Co in Brij 76

Figure 7 shows a linear dependence of $\mathrm{i}_{\mathrm{p}}-\mathrm{v}^{1 / 2}$, and particularly the increase of the current in the peak with the square root of scan rate $\left(\mathrm{v}^{1 / 2}\right)$, indicate that the deposition of Co is a diffusion controlled process. It corresponds to diffusion - controlled process with interference on the chemical stage preceding the reaction of discharge of $\mathrm{Co}^{2+}$ [24]. The shift of the reductive peak potential towards more negative values is a proof supporting the above suggestion.

\subsection{Chronoamperometry Study}

Chronoamperometry is a powerful tool to study the nucleation/growth manner of metals [25]. The i-t transient curves which measured the deposition of the cobalt in lyotropic liquid crystal electrolyte bath are shown in Figure 8. The measured step potential range was from $-0.84 \mathrm{~V}$ to -0.94 V (vs. SCE).

Figure 8 indicates that the current decreases with time as a result of depletion of cobalt ion and is explained with the simultaneous growth mechanism of the nuclei formed. The suggestion that Co ions are involved and is supported by the previous investigations proving their shape and stability [25] that are also comparable with those established in literature [26, 27]. It was found that in slightly acidic lyotropic liquid crystal electrolyte $(\mathrm{pH}=5.2)$, cobalt present as free metal ion $\left(\mathrm{Co}^{2+}\right)$. The general conclusion suggested by the chronoamperometric analysis is that the behavior of the curves in Fig. 8 evidences for a typical process of nucleation with three - dimensional growth of the nuclei at predominantly diffusion control on the part of the electroactive forms [28]. The critical time for nucleation, i.e. the time in which the current decreases with the increase of the applied cathodic potential. It was found that, the method of chronoamperometry provides clear diagnostic criteria to recognize the mechanism of nucleation.

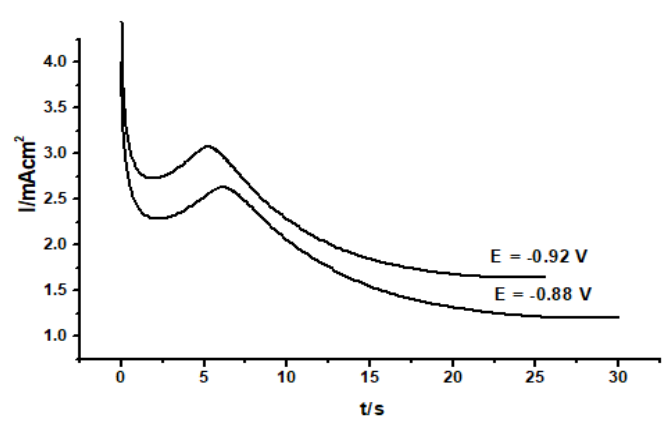

Figure 8. Current densities obtained from chronoamperometry experiments of Cobalt (II)/Brij 76 at different step potentials
Table 1. $I_{m}$ and $t_{m}$ of $I-t$ curves for cobalt deposited at -0.84 V (vs. SCE) to $-0.92 \mathrm{~V}$ (vs. SCE)

\begin{tabular}{ccc}
\hline E/V vs. SCE & $\mathrm{I}_{\mathrm{m}}\left(\mathrm{mAcm}^{-2}\right)$ & $\mathrm{t}_{\mathrm{m}} / \mathrm{s}$ \\
\hline-0.84 & 1.45 & 7.9 \\
-0.86 & 1.73 & 7.2 \\
-0.88 & 1.94 & 6.8 \\
-0.90 & 2.26 & 6.2 \\
-0.92 & 2.64 & 5.8 \\
\hline
\end{tabular}

Table 1 Show the calculated $\mathrm{I}_{\mathrm{m}}$, and $\mathrm{t}_{\mathrm{m}}$, for the Co electrodepositions where the $\mathrm{I}_{\mathrm{m}}$ and $\mathrm{t}_{\mathrm{m}}$ are the maximum peak current in the chronoamperometric diagrams and the time taken to get the peak current. It was noted that the $I_{m}$ increases with increasing the potential step and the $t_{m}$ decreases with increasing the potential step, confirming that the process of electrodeposition of cobalt is attributed to the instantaneous and progressive nucleation mechanism.

According to the theory developed by Scharifker, Hills [28], there are two boundary cases of nucleation with a diffusion controlled growth. At a high rate of nucleation all nuclei are formed spontaneously and the dependence time-current is described by:

$$
\begin{aligned}
& \mathrm{i}(\mathrm{t})=\stackrel{\mathrm{zFD}}{\mathrm{I}-\mathrm{-} \mathrm{-} \mathrm{-}} \mathrm{--}]\left[1-\exp \left(-\mathrm{N}_{\mathrm{o}} \pi k D t\right)\right] \\
& (\pi \mathrm{t})^{1 / 2}
\end{aligned}
$$

where $\mathrm{k}=8 \pi \mathrm{Co}^{\infty} \mathrm{M} / \rho$; D is the diffusion coefficient; $\mathrm{Co}^{\infty}$ is volumetric concentration of $\mathrm{Co}^{2+} ; \mathrm{M}$ is molar mass; $\rho$ is density of the metal; $\mathrm{N}_{\mathrm{o}}$ is the number of spots for nucleation. In this case the chronoampograms are linearized in coordinates $\mathrm{I}-\mathrm{t}^{1 / 2}$. On the other hand i.e. at a lower rate of nucleation, when the nuclei are formed continuously within the entire time window before reaching the area of overlapping with the diffusion limitation around the growing nuclei, we can speak about progressive (continuous) nucleation. In this case, the chronoamperogram are linearized in coordinates $\mathrm{I}^{-\mathrm{t}^{-3 / 2}}$. The course of the current for progressive three- dimensional growth mechanism is described by the following equation:

$$
\mathrm{i}(\mathrm{t})=\frac{\mathrm{zFD}^{1 / 2} \mathrm{c}}{[------]}\left[1-\exp \left(-\mathrm{a} \pi \mathrm{N}_{0} k^{\prime} D t^{2} / 2\right)\right]
$$

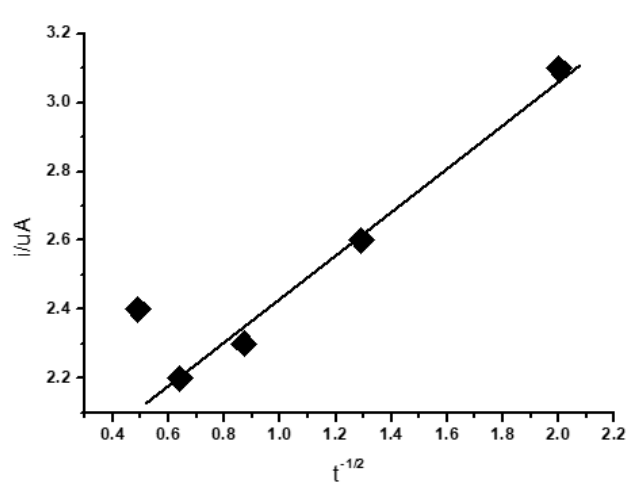

Figure 9. $\mathrm{i}-\mathrm{t}^{-1 / 2}$ plot of Cobalt in lyotropic liquid crystal at gold electrode and step potential $-0.92 \mathrm{~V}$

In the case of $\mathrm{Co}$, we can observe a better linearization of the chronoamperograms coordinates $\mathrm{i}^{-\mathrm{t}^{-1 / 2}}$ (Figure 9), which evidences for a mechanism of spontaneous nucleation that is superimposed onto the general diffusion controlled the process 
of deposition of Co. It is possible that the mentioned difference in the mechanisms of nuclei growth is due to the two-stage transfer of charge of Co since the first stage has already localized the needed spots for crystal growth on the surface. The higher speed of nucleation is in through the mechanism of spontaneous nucleation of Co. It was found that with increasing the applied potential the rate of nucleation increase.

\section{CONCLUSION}

The mesoporous $\mathrm{H}_{1}$-eCo films are successfully electrodeposited on the gold substrate from the hexagonal lyotropic liquid crystalline phase of an inexpensive nonionic surfactant (Brij 76). The obtained results show that the experimental conditions have significant impact on the structure and electrochemical capacitance of the prepared $\mathrm{H}_{1}$ eCo films. The results acquired from TEM and low angle XRD indicate that the presence of Nanostructure and support a direct templating mechanism for the electrodeposition of mesoporous Co films, which implies that the Co films have a higher aspect ratio and excellent mesoporous films display high specific capacitance, which may be attributed to a larger effective surface area within mesoporous films. Chronoamperometric analysis shows that the process of nucleation of cobalt proceeds via three - dimensional growth of the nuclei at predominantly diffusion control on the part of the electroactive forms.

\section{ACKNOWLEDGEMENTS}

This project was supported by King Saud University, Deanship of Scientific Research, College of Science, Research Center.

\section{REFERENCES}

[1] Colvin, V.L., Schlamp, M.C., Alivisatos, A.P. (1994). Light-emitting diodes made from cadmium selenide nanocrystals and a semiconducting polymer. Nature, 370: 354. https://doi.org/10.1038/370354a0

[2] Kesavan, V., Sivanand, S.P., Chandrasekaran, S., Kaltypin, Y., Gedanken, A. (1999). Catalytic Aerobic Oxidation of Cycloalkanes with Nanostructured Amorphous Metals and Alloys. Angewandte Chemie International Edition, 38(23): 3521.

[3] Conway, B.E. (1991). Transition from "Supercapacitor" to "Battery" Behavior in Electrochemical Energy Storage. The Electrochemical Society, 138(6): 1539. https://doi.org/10.1149/1.2085829

[4] Trasatti, S., Kurzweil, P. (1994). Electrochemical supercapacitors as versatile energy stores. Platinum Metals Rev, 38(2): 46-56.

[5] Sarangapani, S., Tilak, B.V., Chen, C.P. (1996). Materials for Electrochemical Capacitors: Theoretical and Experimental Constraints. Journal of The Electrochemical Society, 143(11): 3791.

[6] Andrew, B. (2000). Ultracapacitors: why, how, and where is the technology. Power Source, 91(1): 37-50. https://doi.org/10.1016/S0378-7753(00)00485-7

[7] Zhao, D.D., Xu, M.W., Zhou, W.J., Zhang, J., Li, H.L. (2008). Preparation of ordered mesoporous nickel oxide film electrodes via lyotropic liquid crystal templated electrodeposition route. Electrochim Acta, 53(6): 26992705. https://doi.org/10.1016/j.electacta.2007.07.053

[8] Yoon, S., Lee, J., Hyeon, T., Oh, S.M., Electrochem, J. (2000). Electric double-layer capacitor performance of a new mesoporous Carbon. Journal of The Electrochemical Society, 147(7): 2507. https://doi.org/10.1149/1.1393561

[9] Raman, K.N., Anderson, M.T., Brinker, C.J. (1996). Template-based approaches to the preparation of amorphous, Nanoporous Slilicas. American Chemical Society, 8: 1682. https://doi.org/10.1021/cm960138+

[10] Paavola, A., Ylirusi, J., Rosenberg, J.P. (1998). Controlled release and dura mater permeability of lidocaine and ibuprofen from injectable poloxamer-based gels. Journal of Controlled Release, 52(1-2): 169-178. https://doi.org/10.1016/S0168-3659(97)00206-X

[11] Liu, J., Shin, Y., Nie, Z., Chang, J.H., Wang, L.Q., Fryxell, G.E., Samuels, W.D., Exarhos, G.J. (2000). J. Phys. Chem A, 104: 8328.

[12] Raimondi, M.E. (2001). Liq. Cryst., 26: 305-339. https://doi.org/10.1080/026782999205100

[13] Mitchell, D.J., Tiddy, G.J.T., Waring, J., McDonald, J.T. (1983). Phase behaviour of polyoxyethylene surfactants with water. Mesophase structures and partial miscibility (cloud points). Journal of the chemical society, 79: 975. https://doi.org/10.1039/F19837900975

[14] Bartlett, P.N., Birikin, P.R., Ghanem, M.A., Groot, P.D., Sawicki, M. (2001). The Electrochemical Deposition of Nanostructured Cobalt Films from Lyotropic Liquid Crystalline Media. Journal of The Electrochemical Society, 148: C119. https://doi.org/10.1149/1.1342178

[15] Hongmei, L., Li, S., Yunfeng, L., Yushan, Y. (2004). Langmuir, 20(23): 10218.

[16] El-Hallag, I.S. (2009). Electrochemical and SEM properties of $\mathrm{Co} 2+$ ion in hexagonal mesophase of pluronic lyotropic liquid crystal template. Microfluidics and Nanofluidics, 32: 555.

[17] Wang, L.Z., Shi, J.L., Yu, J., Yan, D.Y. (1998). Synthesis of nanostructured mesoporous silica materials containing manganese. Nanostructure Mater, 10: 1289. https://doi.org/10.1016/S0965-9773(99)00009-4

[18] Karakasside, M.A., Bourlions, A., Petridis, D., CocheGuerente, L., Labbe, P.J (2000). Mater. Chem., 10: 403.

[19] Tan, Y.W., Srinivasan, S., Choi, K.S. (2005). Electrochemical Deposition of Mesoporous Nickel Hydroxide Films from Dilute Surfactant Solutions. Chem. Soc., 127(10): 3596-3604.

[20] Zhou, W.J., Zhao, D.D., Xu, M.W., Xu, C.L., Li, H.L. (2008). Effects of the electrodeposition potential and temperature on the electrochemical capacitance behavior of ordered mesoporous cobalt hydroxide films. Electrochim. Acta, 53(24): 7210-7219.

[21] Lee, M.H., Oh, S.G., Suh, K.D., Kim, D.G., Sohn, D. (2002). Colloid Surf., A210: 49-60.

[22] Cleverson, S., Rafaela, D., Luı's, F., Christiana, A.P., Arab J Chem., 13(1), 3448.

[23] Brown, E.R., Large, R.F. (1971). Cyclic Voltammetry, A.C. Polarography and Related Techniques of Chemistry. In: Brown, E.R. and Large, R.F., Eds., Physical Methods of Chemistry-Electrochemical Methods, Vol. I, II-A, Wiley Interscience, New York, 423.

[24] Galus, Z., Fundamentals of Electrochemical Analysis (Ellis Horwood Series in Analytical Chemistry), 606, New York (1976). 
[25] Ignatova, K., LilovaK, D., Chem, J. (2015). Technol. Metall., 50: 207.

[26] Zelenin, O.Y. (2007). Interaction of the Ni2+ ion with citric acid in an aqueous solution. Russian Journal of Coordination Chem., 33: 346.

[27] Kotsakis, N., Raptopoulou, C.P., Tangoulis, V., Terzis, A., Giapintzakis, J., Jakusch, T. (2003). Correlations of Synthetic, Spectroscopic, Structural, and Speciation
Studies in the Biologically Relevant Cobalt (II)-Citrate System: The Tale of the First Aqueous Dinuclear Cobalt (II)-Citrate Complex. Inorganic Chemistry, 42(1): 22-31. https://doi.org/10.1021/ic0112721

[28] Scharifker, B. (1983). Theoretical and experimental studies of multiple nucleation. Gr. Hills, Electrochimica Acta, 28(7): 879-889 